\title{
7.5.6 Diabetes-Therapie
}

Grundsätzliches Therapieziel beim Diabetes mellitus ist die Optimierung der Blutzuckerwerte. Dies gelingt nur dann, wenn der/die Patientln versteht, dass er/sie als Schlüsselfigur eines Behandlungsteams für die gute Einstellung seines Diabetes mellitus verantwortlich ist. Diabetes-PatientInnen sollten darin geschult sein, den Blutzucker selbst zu messen und - v.a. bei Diabetes mellitus Typ 1 - auch selbst Therapieanpassungen vorzunehmen. PatientInnen mit T1DM müssen zwingend mit Insulin behandelt werden, da es sonst rasch zu schwerwiegenden Stoffwechselentgleisungen kommen kann. Bei PatientInnen mit Diabetes mellitus Typ 2 werden primär Lifestyleänderungen initiiert. Sind diese nicht ausreichend erfolgreich, werden in einem zweiten Schritt orale Antidiabetika wie Metformin oder Sulfonylharnstoffe eingesetzt. Alternative neuere Medikamente aus der Gruppe der Inkretine (GLP-1-Analoga, DPP-4-Inhibitoren) können die Therapie ergänzen. Gelingt es trotz geändertem Lifestyle und oralen Antidiabetika nicht, den $\mathrm{HbA}_{1 \mathrm{c}}$-Wert unter $7 \%$ zu senken, muss oftmals zusätzlich Insulin gegeben werden. Insbesondere beim T2DM ist es wichtig, auch weitere kardiovaskuläre Risikofaktoren wie Bluthochdruck und Dysplidämie optimal zu behandeln.

\section{Internet-Ressourcen}

Auf unserer Lehrbuch-Homepage (www.public-health-kompakt.de) finden Sie Hinweise auf weiterführende Literatur sowie Links zu themenrelevanten Studien und Institutionen.

\subsection{Atemwegserkrankungen und Allergien \\ Claudia Kuehni, Philipp Latzin}

Aufgrund ihrer großen Oberfläche und des dort stattfindenden Gasaustauschs ist die Lunge den zahlreichen Luftschadstoffen unmittelbar ausgesetzt. Besonders empfindlich hierfür ist die noch wachsende Lunge. Atemwegserkrankungen und Allergien können dann die Folge sein. Sowohl in Mitteleuropa wie auch in den sog. Entwicklungsländern sind Atemwegserkrankungen eine bedeutende Ursache der Krankheitslast (Burden of Disease). Betroffen sind vorwiegend Kinder und ältere Menschen. In Entwicklungsländern tragen Lungenentzündung und Tuberkulose signifikant zur Gesamtmortalität bei, in den westlichen Industrienationen sind chronische Atemwegserkrankungen wie COPD und Asthma für einen erheblichen Teil der Gesamtmorbidität verantwortlich.

In diesem Abschnitt betrachten wir die Entwicklung der Lungenfunktion im Laufe des Lebens, die unterschiedliche Vulnerabilität der Lunge gegenüber Umwelteinflüssen in den verschiedenen Entwicklungsstadien sowie die globale Krankheitslast als Folge von respiratorischen Erkrankungen. Anschließend beschreiben wir das Asthma und die COPD hinsichtlich ihrer Risikofaktoren und erörtern, mit welchen präventiven Maßnahmen diese Erkrankungen zu verhindern wären.

Schweizerische Lernziele: $\mathrm{CPH} 40$ 


\subsubsection{Der Respirationstrakt im Laufe des Lebens}

Kinder reagieren besonders empfindlich auf Umwelteinflüsse:

- Im Verhältnis zu ihrem Körpergewicht trinken sie mehr Wasser als Erwachsene, essen mehr Nahrung und atmen mehr Luft ein. Pro $\mathrm{m}^{2}$ Alveolarfläche ventiliert ein Erwachsener z. B. 7,5 ml Luft/min, ein Neugeborenes jedoch 500ml Luft/min. Die Folge ist eine beträchtlich höhere Schadstoffexposition.

- Viele Stoffwechselwege, v.a. Stoffaufnahme (Absorption), Stoffausscheidung (Exkretion) und Entgiftung (Detoxifikation), sind noch unreif, so dass Schadstoffe länger und intensiver auf den Organismus einwirken.

- Die geringere Körpergröße und eine höhere körperliche Aktivität potenzieren zudem die Auswirkungen verkehrsbedingter Luftverschmutzung (so ist z. B. die kindliche Nase näher am Auspuff von Fahrzeugen).

- Insgesamt reagiert das sich noch entwickelnde respiratorische System empfindlicher (vulnerabler) auf Störfaktoren.

Die Vulnerabilität gegenüber Umwelteinflüssen variiert abhängig von der Phase der kindlichen Entwicklung und vom jeweiligen Schadstoff. Es gibt Phasen erhöhter Empfindlichkeit (Windows of Susceptibility), z. B. im Säuglingsalter, wo die Lunge besonders schnell wächst und das Abwehrsystem noch nicht voll ausgebildet ist. Umweltepidemiologische Studien müssen daher immer das Alter der Kinder zum Zeitpunkt der Exposition berücksichtigen.

Lungenentwicklung: Die Lungenentwicklung vollzieht sich in Phasen. Die Organanlage entsteht ab der 5. Gestationswoche (GW). Am Ende der so genannten pseudoglandulären Phase (5.-17. GW) existiert bereits ein vollständiger Bronchialbaum, der sich in den anschließenden Wochen weiter ausbildet. Die Atemwegsentwicklung kann deshalb bereits durch verschiedene pränatale Einflüsse (z.B. mütterliches Rauchen) erheblich beeinflusst werden. Die Entwicklung der Lungenbläschen (Alveolen) beginnt in der 36. GW und erreicht ihr Maximum in den ersten beiden Lebensjahren. Entgegen früherer Annahmen geht die Entwicklung der Alveolen mit verminderter Intensität wahrscheinlich bis zum Abschluss des Lungenwachstums im frühen Erwachsenenalter weiter. Während des gesamten Wachstums besteht deshalb eine erhöhte Vulnerabilität gegenüber Umwelteinflüssen, vermutlich aber auch ein erhöhtes Potenzial zur Durchführung von Reparaturen.

Lungenfunktion: Die Lungengröße nimmt während der Kindheit und Jugend bis zum Erreichen des individuellen Lungenfunktions-Höchstwertes im Alter von etwa 18 (ㅇ) bzw. $20(\hat{\jmath})$ Jahren kontinuierlich zu. Danach beginnt die Lungenalterung, gekennzeichnet durch einen andauernden Abfall der Lungenfunktionswerte bis zum Lebensende (Abb. 7.6). Wegen der großen funktionellen Reserve treten Symptome, wie z. B. Kurzatmigkeit, erst nach dem Unterschreiten eines Schwellenwertes auf. Wann dieser Zeitpunkt individuell erreicht wird, hängt vom Ausmaß des Anstiegs während der Wachstumszeit, dem jeweiligen Maximum im jungen Erwachsenenalter und von der Steilheit des Abfalls ab.

Einflüsse auf die wachsende Lunge: Einflüsse auf die wachsende Lunge während der Schwangerschaft und der frühen Kindheit (z.B. die intrauterine/postnatale Ernährung, 


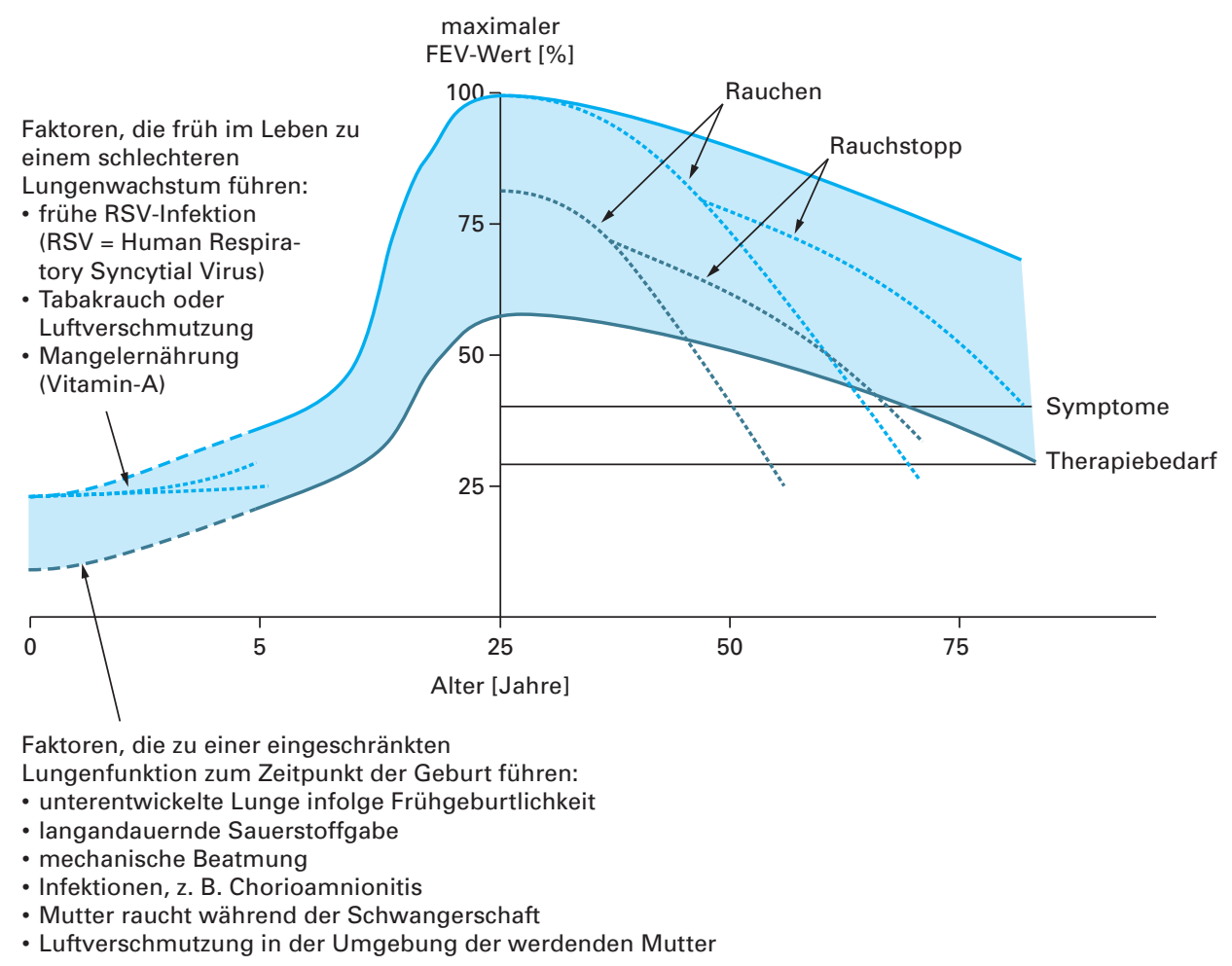

Abb. 7.6: Schematische Darstellung der Lungenfunktion im Lauf des Lebens (Quelle: modifiziert nach Baraldi E, Filippone M. Chronic Lung Disease after Premature Birth. The New England Journal of Medicine 2007; 357: 1946-1955).

Tabakrauchexposition, Luftverschmutzung, Asthma oder Atemwegsinfekte) beinträchtigen das Lungenwachstum und damit das individuelle Maximum der Lungenfunktion. Hierbei ist insbesondere das Zusammenspiel zwischen Lungenwachstum und Entwicklung des Immunsystems von Bedeutung, da beide Faktoren z. B. bei der Entstehung von Asthma eine entscheidende Rolle spielen.

Einflüsse auf die reife Lunge: Einflüsse auf die reife Lunge im Erwachsenenalter (z. B. Aktiv- und Passivrauchen, Luftverschmutzung oder Exposition am Arbeitsplatz) beeinflussen die Steilheit des Abfalls der Lungenfunktion. Sowohl ein niedriges Lungenfunktionsmaximum als auch ein steilerer Abfall bewirken, dass die symptomatische Schwelle früher erreicht wird. Nach der Beendigung einer schädlichen Exposition (z.B. durch Rauchstopp) können sich das Lungenwachstum bei Kindern und die Geschwindigkeit des Lungenfunktionsabfalls bei Erwachsenen wieder normalisieren (Abb. 7.6.). Der Umfang ist dabei stark abhängig von der Dauer und der Intensität der vorherigen Belastung.

Tracking: Verschiedene Kohortenstudien konnten eine enge Korrelation zwischen der Lungenfunktion im Kindesalter und im späteren Leben zeigen. Diesen Zusammenhang bezeichnet man als „Tracking”. Ebenso wie beim Körpergewicht unterstreicht das Tra- 
cking auch bei der Lungenfunktion die Bedeutung der genetischen, epigenetischen, pränatalen und frühkindlichen Einflüsse auf die spätere Entwicklung eines Kindes. (s. "Fetal Programming" oder "Barkers Hypothese"; Näheres dazu finden Sie auf unserer Lehrbuch-Homepage).

Konzept der „United Airways“: Erkrankungen der oberen und unteren Atemwege sind eng miteinander verbunden (Konzept der "United Airways"). So können ein Heuschnupfen oder eine chronische Sinusitis die Entstehung von unteren Atemwegsproblemen (Asthma, chronische Bronchitis) bahnen. Beteiligt hieran sind verschiedene Mechanismen, wie z. B. die fehlende natürliche Reinigung durch die Haare der Nase bei Mundatmung, Sekretfluss aus den oberen in die unteren Atemwege oder die systemische Verbreitung von zuvor lokal gebildeten Entzündungsmediatoren. Die einzelnen Schadstoffe wirken dabei unterschiedlich auf die verschiedenen "Atemwegs-Etagen": grobe Partikel und größere Aerosole werden im oberen, feinere Partikel und kleinere Aerosole im unteren Atmungstrakt deponiert.

\subsubsection{Epidemiologie der Erkrankungen der Atemwege}

Unter den 20 weltweit häufigsten Todesursachen listet die WHO (2004) vier respiratorische Erkrankungen auf.

- Untere Atemwegsinfekte (uAWI) sind nach den ischämischen Herzerkrankungen die zweitwichtigste Todesursache: 4,1 Mio. Tote/Jahr entsprechen 7\% aller Todesfälle weltweit.

- Die chronisch obstruktive Lungenerkrankung (COPD) nimmt den 3. Platz ein: 3 Mio. Tote/Jahr entsprechen $5 \%$ aller Todesfälle.

- Die Tuberkulose (Tbc) liegt auf Platz 7: 1,5 Mio. Tote/Jahr entsprechen 2,5\% aller Todesfälle,

- gefolgt vom Lungenkrebs auf Platz 8: 1,3 Mio. Tote/Jahr entsprechen 2,3\% aller Todesfälle.

In den weniger entwickelten Ländern nehmen die uAWI sogar den ersten Platz bei den häufigsten Todesursachen ein. Auch zur globalen Krankheitslast (Burden of Disease, ausgedrückt in Disability-Adjusted Life Years, DALYs, s. Kap. 9.1.2) tragen die respiratorischen Erkrankungen in erheblichem Umfang bei. Hier liegen die uAWI mit 94 Mio. DALYs (6\%) auf Platz 1, die Tbc mit 34 Mio. DALYs (2\%) auf Platz 11 und die COPD mit 30 Mio. DALYs (2\%) auf Platz 13. In der Schweiz und in Deutschland sind Atemwegsinfekte und Asthma bei Kindern häufige Gründe für Arztbesuche und Krankenhausaufenthalte. Durch den Erwerbsausfall der Eltern kranker Kinder entstehen zusätzlich beträchtliche indirekte Kosten.

\subsubsection{Risikofaktoren für Atemwegserkrankungen}

Risikofaktoren für Atemwegsinfekte sind einerseits Faktoren, die die Exposition gegenüber Infektionserregern erhöhen, wie z. B. die Anzahl der Geschwister oder eine Kinderbetreuung in großen Gruppen (Kindertagesstätten). Andererseits spielen individuelle Faktoren der Betroffenen eine wichtige Rolle. Dies können Faktoren sein, die die Im- 
munabwehr (Impfungen, Stillen, Mangelernährung), die Lunge direkt (Innen- und Außenluftverschmutzung, Asthma, Allergien) oder beides (genetische Veranlagung, Rauchen während der Schwangerschaft) beeinflussen.

Risikofaktoren für chronische Atemwegserkrankungen wie Asthma, COPD und Lungenkrebs entstehen mehrheitlich durch menschliches Verhalten und sind damit oft vermeidbar. So ist Tabakrauch weltweit für 8,7\% der Gesamtmortalität und für 3,7\% der verlorenen Lebensjahre (ausgedrückt in DALYs) verantwortlich. Die meist durch Holzfeuerung hervorgerufene Luftbelastung in Innenräumen führt zu 3,3\% der globalen Gesamtmortalität und zu 2,7\% der in DALYs angegebenen Krankheitslast. Weitere Risikofaktoren sind Außenluftverschmutzung und berufliche Luftschadstoff-Exposition (s.a. Kap. 6.2, Kap. 7.1, Kap. 7.2).

\subsubsection{Asthma und COPD als Beispiele chronischer Atemwegserkrankungen}

Asthma und Allergien

Asthma zählt zusammen mit Heuschnupfen und Neurodermitis zu den atopischen Erkrankungen. Allerdings ist nur ein Teil der Asthmaerkrankungen auf allergische Reaktionen zurückzuführen („Attributable to Atopy“). In den entwickelten Ländern sind es ca. $50 \%$, in den weniger entwickelten Ländern nur etwa $25 \%$. Vieles weist darauf hin, dass sich unter dem Syndrom „Asthma“ verschiedene Krankheitsentitäten verbergen, die durch unterschiedliche Ätiologie, Pathophysiologie, therapeutisches Ansprechen und Prognose gekennzeichnet sind. Bestimmte Asthma-Phänotypen gehen mit einem erhöhten COPD-Risiko im Alter einher.

Diagnose: Die Diagnose wird primär anhand anamnestischer und klinischer Kriterien gestellt. Zusätzliche Untersuchungen wie Lungenfunktions- und Allergietests, ausgeatmetes Stickoxid (FeNO) oder Provokationstests tragen zur Bestätigung der Diagnose oder zur besseren Charakterisierung des Asthma-Phänotypen bei. Sie erlauben aber selten den Ausschluss eines Asthmas bei entsprechender klinischer Symptomatik. Erschwerend für die Diagnosestellung sind der unterschiedliche Schweregrad der Erkrankung mit fließendem Übergang zum Normalen und die zeitliche Fluktuation mit oft langen asymptomatischen Phasen. Für die epidemiologische Forschung sind deshalb pragmatische Definitionen wichtig. Meist wird die Jahres-Prävalenz von "Current Wheeze" („pfeifende Atmung in den vergangenen 12 Monaten“) erfasst.

Epidemiologische Daten: Dank guter Behandlungsmöglichkeiten ist die Asthma-Mortalität in Mitteleuropa gering. Achtzig Prozent der Todesfälle ereignen sich in Ländern mit mittlerem und niedrigem Einkommen. Mit geschätzten 235 Mio. Asthmakranken (WHO) weltweit trägt die Morbidität dagegen erheblich zur globalen Krankheitslast bei. Im Kindesalter ist Asthma die wichtigste chronische Erkrankung. Die ISAAC-Studie (International Study of Asthma and Allergies in Childhood), die auf die Daten von 2 Mio. Kindern aus über 100 Ländern zurückgreift, belegt die große regionale Variabilität. Hiernach betrug die Prävalenz von "Current Wheeze" in den letzten 10 Jahren in englischsprachigen Ländern (Australien, Neuseeland, England, USA) über 20\%, in Mitteleuropa etwa $10 \%$, in Osteuropa und Ostasien dagegen nur wenige Prozent. Kohortenstudien mit wiederholten Befragungen zeigen, dass im Verlauf der Kindheit bis zu 50\% aller Kinder in Europa mindestens einmal unter Asthma-Symptomen leiden. Der Lang- 
zeitverlauf ist sehr variabel. Nur wenige Kinder haben jahrelang Symptome, bei der Mehrzahl der Kinder treten Symptome nur zeitweise („intermittent") oder vorübergehend („transient") auf.

Zeitliche Veränderungen: Nach einer starken Zunahme der Asthma-Prävalenz im vergangenen Jahrhundert scheint derzeit eine Stabilisierung eingetreten zu sein. Die beobachtete Zunahme konnte in den letzten Jahren durch serielle Untersuchungen von objektivierbaren asthma-assoziierten Merkmalen wie Atopie und bronchialer Hyperreaktivität zum Teil belegt werden. Inwiefern eine verbesserte Diagnosestellung und erhöhte öffentliche Aufmerksamkeit ebenfalls dazu beitrugen, ist noch unklar.

Risikofaktoren und Prävention: Asthma und Atopien sind multifaktoriell bedingte Erkrankungen. Neben einer genetischen Veranlagung beeinflussen eine Vielzahl von Umweltfaktoren die Entwicklung von Immunsystem und Lunge. Trotz großer Forschungsanstrengungen sind die Ursachen der regionalen Variabilität und der zeitlichen Trends nur ungenügend bekannt. Eindeutige Risikofaktoren sind prä- und postnatale Tabakrauchexposition und Luftverschmutzung. Stillen führt bei Säuglingen zu einem gewissen immunologischen Schutz vor infektassoziiertem Asthma. Ein möglicher positiver Einfluss auf die Lungenentwicklung und die Entstehung von Allergien ist noch nicht eindeutig nachgewiesen. Eine frühe Infektexposition (durch ältere Geschwister, Besuch einer Kinderkrippe) erhöht die Häufigkeit von Infektasthma bei Kleinkindern, führt möglicherweise jedoch zu einer Reduktion der Symptomatik im Schulalter. Einflüsse durch Haustierhaltung, Ernährung, häufige Antibiotikagabe und „bäuerliche Lebensweise" werden derzeit intensiv erforscht, die Resultate sind jedoch bisher nicht eindeutig.

Als Präventionsmaßnahmen werden neben dem Stillen und einer gesunden Ernährung vor allem der Schutz vor Passivrauchen sowie vor Innen- und Außenluftverschmutzung empfohlen. Bei bereits vorhandenen Atemwegserkrankungen sollte die Exposition gegenüber Allergenen reduziert werden. Eine konsequente Therapie führt bei der Mehrzahl der Betroffenen zu Symptomfreiheit und zu einer Reduktion von Exazerbationen (plötzliche Verschlechterungen des Krankheitsbildes). Allerdings lassen sich mit den derzeit vorhandenen Therapien bisher weder die Langzeitprognose positiv beeinflussen noch eine Heilung erreichen.

\section{Chronisch obstruktive Lungenerkrankung (COPD)}

Ähnlich wie beim Asthma wurde die COPD-Forschung durch eine unklare Terminologie und das Fehlen von akzeptierten diagnostischen Standards erschwert. Die Global Initiative for Chronic Obstructive Lung Disease (GOLD) hat inzwischen zu einer Standardisierung der Kriterien beigetragen. In epidemiologischen Studien wird der Begriff COPD heute meist funktionell anhand des Verhältnisses von forcierter Einsekundenkapazität (FEV1) zu forcierter Vitalkapazität (FVC) definiert. Hiernach spricht man von einer COPD, wenn die FEV1/FVC-Ratio nach Bronchodilatation (medikamentöser Erweiterung der Bronchien) kleiner als 0,7 ist.

Epidemiologische Daten: Je nach der gewählten Definition können die Schätzungen zur COPD-Prävalenz erheblich variieren. Die meisten epidemiologischen Daten zu Prävalenz, Morbidität und Mortalität der COPD stammen aus entwickelten Ländern. 
Nach groben Schätzungen leidet weltweit mindestens $1 \%$ der Gesamtbevölkerung an COPD. Die Häufigkeit ist altersabhängig, von den über 40-Jährigen sind 10 bis $15 \%$ betroffen. Nach Angaben der WHO gibt es weltweit ca. 80 Mio. Patienten mit mittelschwerer oder schwerer COPD. Pro Jahr fallen der Erkrankung 3 Mio. Menschen zum Opfer, 90\% davon in den weniger entwickelten Ländern. Wenn sich die Risikofaktoren der COPD wie Tabakrauchen und Innen- bzw. Außenluftverschmutzung bis zum Jahr 2020 nicht drastisch reduzieren lassen, wird die Prävalenz um weitere $30 \%$ zunehmen. Bis zum Jahr 2030 wird die COPD weltweit die dritthäufigste Todesursache sein.

Für die Schweiz und Deutschland gibt es keine detaillierten epidemiologischen Daten zur COPD. Die bevölkerungsbasierte SAPALDIA-Kohortenstudie aus der Schweiz beobachtete 1992 bei 18- bis 60-jährigen Bewohnern eine durchschnittliche COPD-Prävalenz von 9,1\%. Davon hatten $85 \%$ eine leichte COPD (GOLD Stadium 1), 57\% hiervon waren asymptomatisch. Bis zum Jahr 2003 entwickelten weitere 14\% der erwachsenen Bevölkerung eine COPD. Dies entsprach einer Inzidenz von 1,3\% pro Jahr.

Risikofaktoren und Prävention: Der mit Abstand bedeutendste Risikofaktor für die COPD ist das Rauchen, gefolgt von Innen- und Außenluftverschmutzung sowie beruflicher Luftschadstoff-Exposition. Frühgeburtlichkeit und frühkindliche Infekte beeinflussen nicht nur das Lungenwachstum, sondern korrelieren auch mit der COPD-Mortalität. War die COPD früher eine "Männerkrankheit", so ist die Prävalenz heute bei beiden Geschlechtern ähnlich. Hauptursache hierfür ist die abnehmende Zahl an Rauchern und die Zunahme an Raucherinnen in den westlichen Industrienationen. Darüber hinaus sind Frauen in den weniger entwickelten Ländern durch das Verbrennen von Biomasse zum Kochen und Heizen in Innenräumen häufiger einer Innenraumluftverschmutzung ausgesetzt.

Etwa die Hälfte der COPD-Erkrankungen werden dem Rauchen zugeschrieben. Bis zu $50 \%$ der chronischen Raucher entwickeln eine COPD. Man schätzt, dass es in der Schweiz hierdurch zu 1.300 Todesfällen pro Jahr kommt. Durch einen Rauchstopp lässt sich die Steilheit des Lungenfunktionsabfalls bei den Betroffenen jedoch wieder normalisieren und die COPD-Progression stoppen (s. Abb. 7.6).

\section{Internet-Ressourcen}

Auf unserer Lehrbuch-Homepage (www.public-health-kompakt.de) finden Sie Hinweise auf weiterführende Literatur zusätzliche Abbildungen sowie Links zu den erwähnten Studien (ISAAC, SAPALDIA) und Institutionen. 